\title{
Modelo predictivo de la intención emprendedora universitaria en Latinoamérica
}

\author{
Catherine Krauss Delorme ${ }^{1 *}$ Adriana Bonomo Odizzio ${ }^{1}$, Roberto Volfovicz León ${ }^{1}$
}

Resumen: El presente estudio explora la intención emprendedora y los factores que inciden en la misma, para estudiantes universitarios de seis países latinoamericanos provenientes de empresas familiares. Se trabaja con un modelo de regresión ordinal logística con el fin de probar las hipótesis del modelo teórico de investigación y generar una ecuación matemática que permita cuantificar y predecir la intención emprendedora de un estudiante universitario utilizando como variables explicativas: el tipo de relación con la empresa familiar, la vinculación con la innovación, la motivación para emprender, la actitud emprendedora (entendida como búsqueda de placer y poder) y el PBI de cada país. La investigación fue realizada en el marco RLIE (Red Latinoamericana de Innovación y Emprendimiento) en estudiantes que por lo menos tenían un curso de emprendedurismo en su carrera del área empresarial o ingeniería y provenían de nueve universidades diferentes de América Latina.

Palabras clave: Empresa familiar; Intención emprendedora; Emprendimiento universitario.

\begin{abstract}
Predictive Model of University Entrepreneurial Intention in Latin America
The present study explores the entrepreneurial intention and the factors that affect in college students from six Latin American countries coming from family businesses. A logistic ordinal regression model is used in order to test the hypothesis from the theorical model of investigation, and to generate a mathematical equation that allows to quantify and predict the entrepreneurial intention in college students using as explanatory variables: the type of relationship with the family business, the link with innovation, the motivation to undertake, the entrepreneurial attitude (understood as a search for leisure and power) and the GDP of each country The investigation was made in the frame of CLADEA'S RLIE (Red Latinoamericana de Innovación y Emprendimiento de CLADEA) in students that had at least one entrepreneurship course in their career in the area of business or engineering and came from one of nine different Latin American universities.
\end{abstract}

Keywords: Family Business; Entrepreneurial intention; University entrepreneurship

Submitted: Julyt $16^{\text {th }}, 2018$ / Approved: November $6^{\text {th }}, 2018$

\section{Introducción}

El estudio busca identificar factores que ayudan a predecir la intención emprendedora del estudiante universitario en América Latina. Los emprendedores tiene un rol fundamental en la creación de bienestar en la sociedad, impactando de forma positiva en el desarrollo de las economías de los países, por lo que es importante el éxito en el aprendizaje del espíritu empresarial innovador (Fayolle, 2013; Kakouris y Georgiadis, 2016).

Este trabajo fue posible gracias a la colaboración de universidades participantes de la Red Latinoamericana de Innovación y Emprendimiento (RLIE) que permitió recabar 3.341 datos de estudiantes de México, Puerto Rico, Ecuador, Colombia, Brasil, Uruguay y Chile, provenientes de nueve universidades que buscan promover el emprendimiento entre sus estudiantes. Para esta investigación se seleccionó una submuestra de 771 universitarios que provenían de empresas familiares y ocho universidades.

Para explicar la intención emprendedora, los factores analizados fueron: el tipo de relación con la empresa familiar, la vinculación con la innovación, la motivación para emprender, y la actitud emprendedora, entendida como búsqueda de placer y poder (Bonomo, Krauss, \& Volfovicz, 2017). Por otra parte, las variables de control utilizadas fueron el PBI de los países y una división Norte-Sur que se obtuvo en una investigación anterior realizada por este equipo y publicada en CLADEA 2016 (Krauss, y otros, 2016).

El aporte realizado es relevante ya que se genera una ecuación matemática predictora de la intención emprendedora de un estudiante universitario, aplicando un modelo de regresión ordinal logística a nivel latinoamericano que puede ser utilizado por académicos que quieran profundizar aspectos en la investigación, por las empresas familiares para que puedan contribuir a detectar el interés del hijo/a en dirigir la empresa a futuro y apoyarlo en la formación tanto interna como a nivel de la elección de su carrera, y para stakeholders a los que les preocupe el desarrollo de la actividad emprendedora y el negocio familiar.

El presente documento se inicia con una revisión de literatura en temas relacionados con el emprendimiento. A continuación se describe la metodología utilizada para desarrollar la investigación así como los resultados obtenidos. Por último se desarrollan comentarios finales, limitaciones del estudio y futuras investigaciones.

La teoría del comportamiento planificado futuro (TPB- Theory of Planned Behavior) de Ajzen (1991) sirve de marco de referencia para investigaciones ya que la intención es considerada un indicador del grado de esfuerzo individual o grupal y de la disposición a ejecutar un comportamiento cuando sea el momento y la oportunidad

(1) Departamento de Administración, Universidad Católica del Uruguay, Uruguay.

^Autor de correspondencia: ckrauss@ucu.edu.uy 
adecuada. Los tres factores que explican el comportamiento son: 1) la evaluación personal, 2) la presión social y 3) la facilidad o dificultad percibida para su realización.

El cuestionario en el que se basó la investigación utilizó en TBP ya que es una herramienta con un fuerte poder para llegar a pronosticar la intención emprendedora de los estudiantes universitarios (Krueger, Reilly y Carsrud, 2000; Liñán y Chen, 2009; Maes, Leroy, y Sels, 2014). A pesar de esto, se han encontrado evidencias que el hecho de que los estudiantes indiquen que tienen intención de emprender no implica que luego lo lleven a cabo (Shirokova, Osiyevskyy, y Bogatyreva, 2016).

Por otra parte, se ha analizado el impacto positivo en la intención emprendedora de los estudiantes luego de realizar cursos de emprendimiento a nivel universitario, dónde desarrollan la creatividad, la innovación en las ideas y la generación de modelos de negocios, mostrando luego un mayor deseo de iniciar su propio emprendimiento (Bergmann, Hundt, y Sternberg, 2016).

Uniendo la TBP con estudios universitarios, se ha encontrado que el contexto familiar y de amigos tiene una mayor influencia en la intención de emprender que el entorno de la universidad (Yurtkoru, Kuscu, y Doganay, 2014). Por lo que el desarrollo del espíritu emprendedor tiene una estrecha relación con proceder de una familia empresarial, siendo la motivación una estrategia clave y un antecedente importantes de la intención emprendedora (GUESSS, 2013).

A su vez, los factores individuales como rasgos de personalidad, actitudes y el contexto institucional condicionan las intenciones emprendedoras de los estudiantes entre 18 y 34 años (Gubik y Farkas, 2016). El GEM (Global Entrepreneurship Monitor) a nivel mundial ha encontrado que los jóvenes tienen 1,6 veces mayor intención emprendedora que los adultos (Schott, Kew, y Cheraghi, 2015).

Dentro de los factores analizados en esta investigación se encuentra la relación de los estudiantes con la empresa familiar, entendiendo la misma como un negocio gobernado y/o manejado con la intención de formar y seguir la visión de la empresa por una coalición dominante, controlada por los miembros de una misma familia o de una pequeña cantidad de familias de una manera que es potencialmente sostenible a través de las generaciones (Navarro de Granadillo, 2008).

La visión compartida por los miembros de la empresa familiar tiene un gran efecto en el grado de involucramiento de los jóvenes con su trabajo como miembros del equipo (Miller S. P., 2014). Por otra parte, considera que solamente es positivo el efecto si la experiencia del modelo parental es exitosa (Criaco, Sieger, Wennberg, Chirico, y Minola, 2017).

Las empresas familiares tienen un rol dominante en el desarrollo económico de muchos países, pero presentan dificultades a la hora de la sucesión familiar, impactando en el futuro del negocio (Luan, Chen, Huang, y Wang, 2017). Brännback, Carsrud y Schulte (2008) identifican, en su estudio del proceso de sucesión de la empresa familiar, la importancia de la percepción del lugar que ocupa como miembro de la familia, así como lo esencial de la creación de conocimiento y el compartirlo. A su vez, el aspecto que más influye en el deseo de dirigir la empresa familiar es haber elegido una carrera profesional relacionada con la actividad principal de la empresa (González y Herrera, 2017).

Otro factor de la investigación es cuantificar cómo la innovación que realizan las empresas impacta en la motivación que tienen los estudiantes por trabajar en ellas. Para ello es fundamental la postura que existe en la empresa familiar respecto a la innovación. La misma se potencia cuando muchos de los miembros están a favor, o es perjudicada por una mala relación familiar. La elección de un sucesor competente y no elegido simplemente por favoritismo del fundador, augura un éxito en el esfuerzo innovador (Rondi, De Massis, y Kotlar, 2017).

La familia debe estar activamente involucrada en el management para mejorar el ratio de conversión de aplicación de la innovación. Solamente contar con personal capacitado y experiente no alcanza si no hay una gerencia profesional tanto externa como familiar (DiéguezSoto, Garrido-Moreno, y Manzaneque, 2018). La innovación está muy influencia por la interdependencia entre diferentes tipos de contexto: público, político, organizacional, social, sectorial, financiero, el financiamiento, y políticas públicas (Autio, Kenney, Mustar, Siegel, y Wright, 2014).

En el cuestionario utilizado, los factores de motivación y actitud personal se analizan en conjunto. En la teoría del TBP, el control interno percibido tiene que ver con la autoeficacia, autodirección y el componmente de contexto social en el control percibido externo. La autoeficacia puede ser determinada preguntando las percepciones individuales de ser capaces de realizar un determinado comportamiento Por otra parte, el control gira en cómo alguien se siente en el dominio de la performance (o no performance) del comportamiento. Ambos conceptos, autoeficacia y control, están interrelacionados y se usan como el constructo de control percibido interno en la identidad social del emprendedor (Ajzen I. , 1991; Ajzen I. , 2002; Brändle, Berger y Kuckertz, 2018).

McClelland (1973) recalca el impacto de la necesidad de logro (nlogro) y la necesidad de poder (n-poder) en los emprendedores. El desarrollo de las competencias profesionales es importante para la educación de las futuras generaciones y es esperable que contribuya positivamente en los estudiantes en temas de seguridad en sí mismos, competencias y características emprendedoras (Karabulut y Dogan, 2018).

Si bien nuestro análisis de componentes principales no coincidió en todos los aspectos de la teoría, en este estudio se consideraron varios factores de TBP, como la relación que tienen los estudiantes con la empresa familiar, la innovación realizada en la misma, su motivación para emprender y su actitud incorporando además un análisis de cluster considerando las universidades de los países latinoamericanos que participaron de la investigación. 


\section{Metodología}

\section{Muestra}

Se estudió una muestra constituida por 771 estudiantes de 8 Universidades latinoamericanas pertenecientes a la RLIE, de 6 países (EICO Universidad de Valparaíso, Chile; UCU, Universidad Católica del Uruguay, Uruguay; IFRS Instituto Federal de Educação Ciência e Tecnologia do Rio Grande do Sul, Brasil; ESPOL Escuela Superior Politécnica del Litoral, Ecuador; UCSG Universidad Católica Santiago de Guayaquil, Ecuador; TEC Tecnológico de Monterrey en Puebla; México; UNL Universidad Nueva León, México; UMNG Universidad Militar Nueva Granada, Colombia; UIPR Universidad Interamericana de Puerto Rico Recinto Bayamon, Puerto Rico). En el análisis se descartan los datos de Puerto Rico por presentar pocos casos de empresas familiares

\section{Análisis Estadístico}

El análisis estadístico se ejecutó en dos etapas. En una primera fase se procedió a realizar un Análisis de Componentes Principales (ACP). En la segunda fase, los factores extraídos mediante ACP fueron utilizados como variables explicativas (independientes) en el desarrollo e implementación de un modelo de regresión logística ordenada que busca: i) probar las hipótesis del modelo teórico de investigación desarrollado en la fase 1. ii) identificar los factores que significativamente contribuyen al emprendedurismo. iii) llegar a predecir la intención emprendedora (variable explicada o dependiente) de los estudiantes universitarios en el conjunto de universidades latinoamericanas que conforman el universo de estudio. Para el análisis estadistico se utilizó el paquete SPSS V.20

\section{Resultados y Discusión}

Se realizó un ACP con una una rotación ortogonal de tipo Varimax. Se comprueba una muy buena adecuación de los datos al modelo ACP (estadístico KMO Kaiser-Meyer-Olkin = 0,923). Se comprueba además la idoneidad del ACP mediante la prueba de esfericidad de Bartlett (Chi-cuadrado $=9658,739 ; \mathrm{gl}=253$; valor $\mathrm{p}=, 0000$ ). No se detectaron problemas en la matriz de correlaciones ni en la matriz anti-imagen en este estudio.

La Tabla 1 permite decidir el número final de componentes, y se opta por seleccionar los primeros 4 componentes principales que explican aproximadamente el $62 \%$ de la varianza total.

Tabla 1 Varianza total explicada

\begin{tabular}{|c|r|r|r|}
\hline \multirow{2}{*}{ Componente } & \multicolumn{3}{|c|}{ Autovalores iniciales } \\
\cline { 2 - 4 } & Total & \% de varianza & \% acumulado \\
\hline 1 & $\mathbf{7 , 7 0 6}$ & $\mathbf{3 3 , 5 0 5}$ & $\mathbf{3 3 , 5 0 5}$ \\
2 & $\mathbf{3 , 5 7 9}$ & $\mathbf{1 5 , 5 5 9}$ & $\mathbf{4 9 , 0 6 5}$ \\
3 & $\mathbf{1 , 7 1 1}$ & $\mathbf{7 , 4 3 7}$ & $\mathbf{5 6 , 5 0 2}$ \\
4 & $\mathbf{1 , 2 3 8}$ & $\mathbf{5 , 3 8 4}$ & $\mathbf{6 1 , 8 8 6}$ \\
5 & 1,058 & 4,602 & 66,487 \\
\hline
\end{tabular}

Figura 1 Gráfico de sedimentación

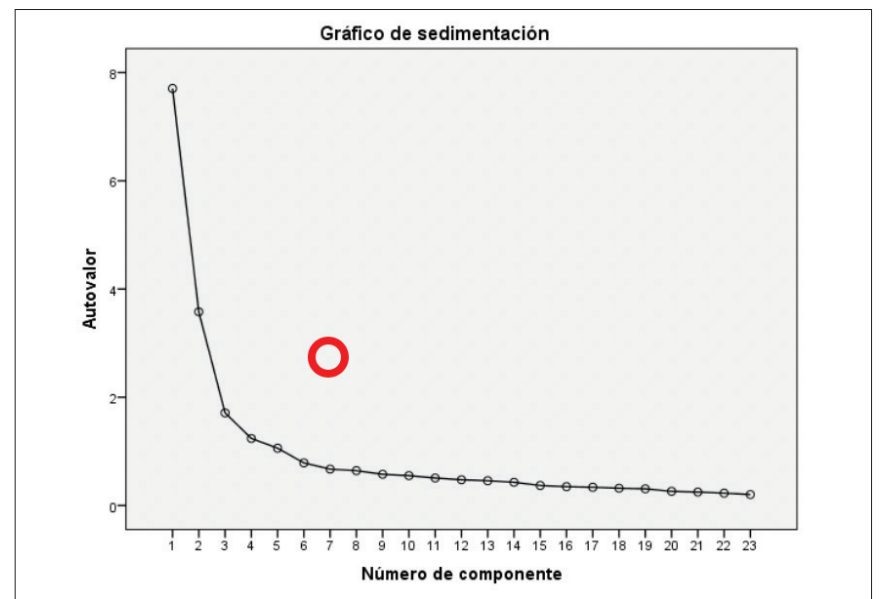

En la Figura 1 se presenta el gráfico de sedimentación de las componentes, el que sugiere la selección de los primeros cuatro factores. Para conseguir una interpretación clara de los factores extraídos se procedió a la rotación factorial Varimax. A continuación, se renombran los componentes en función de las variables iniciales que incorporan:

FACTOR 1. Esta componente engloba al conjunto de atributos que se concentran en la encuesta como pertenecientes al grupo "Relacionamiento con la Empresa”. Este factor explica por sí mismo casi la tercera parte $(33,5 \%)$ de la varianza total (Tabla 1$)$.

FACTOR 2. Esta componente contiene seis variables que marcan la inclinación a la innovación por parte del estudiante. Llamaremos a esta componente el factor de "Innovación", el cual explica casi el 16\% de la varianza total (Tabla 1 ).

FACTOR 3. Incluye seis características que pueden denominarse "Motivación para Emprender". Este componente explica aproximadamente el 7\% de la varianza total. (Tabla 1).

FACTOR 4. Dos variables conforman este componente: 'El logro’ y 'El placer'. Se llamará a este factor: “Actitud Personal: Poder-Hedonismo", explicando casi el 5\% de la varianza total (Tabla 1).

A partir de los factores extraídos por ACP se propone el siguiente modelo teórico de investigación:

\section{Modelo teórico de investigación}

Figura 2 Modelo de la Investigación

\begin{tabular}{|c|c|c|}
\hline $\begin{array}{c}\text { FACTOR } 1 \\
\text { Relacionamiento con la empre }\end{array}$ & & \\
\hline $\begin{array}{l}\text { FACTOR } 2 \\
\text { Innovación }\end{array}$ & \multirow[b]{2}{*}{$\mathbf{H}_{3}$} & \multirow{2}{*}{$\begin{array}{c}\text { Intención } \\
\text { Emprendedora }\end{array}$} \\
\hline $\begin{array}{c}\text { FACTOR } 3 \\
\text { Motivación para emprender }\end{array}$ & & \\
\hline $\begin{array}{c}\text { FACTOR } 4 \\
\text { Actitudes personales: } \\
\text { Poder hedonismo }\end{array}$ & $\mathbf{H}_{4}$ & \\
\hline
\end{tabular}


Las cuatro hipótesis del modelo teórico de investigación son:

$\mathrm{H}_{1}$ : "El relacionamiento con la empresa tiene un efecto positivo en la intención emprendedora de los estudiantes universitarios"

$\mathrm{H}_{2}$ : "La Innovación tiene un efecto positivo en la intención emprendedora"

$\mathrm{H}_{3}$ : "La motivación para emprender tiene un efecto positivo en la intención emprendedora"

$\mathrm{H}_{4}$ : "Las actitudes personales (Poder/Hedonismo) tienen un efecto positivo en la intención emprendedora"

Estas hipótesis fueron estadísticamente testeadas mediante la construcción y utilización de un modelo de regresión logística ordenada cuya variable explicada fue la intención emprendedora de los estudiantes universitarios y cuyas variables explicativas fueron las puntuaciones factoriales de los 4 componentes seleccionados por ACP.

El conjunto de los 4 factores obtenidos del ACP son utilizados como insumo en un modelo de regresión ordinal logística con el fin de probar las hipótesis del modelo teórico de investigación y generar una ecuación matemática que permita predecir la intención emprendedora de un estudiante universitario a partir de esos cuatro factores y variables de control.

La variable dependiente (explicada) es Intención Emprendedora y las independientes son: FACTOR 1 ("Relacionamiento con la Empresa"), FACTOR 2 ("Innovación"), FACTOR 3 ("Motivación para Emprender"), FACTOR 4 (“Actitud Personal: Poder-Hedonismo”), REGIÓN, PIB (Producto Interno Bruto del país).
Estas variables se detallan a continuación:

\section{- Variable dependiente}

Intención emprendedora del estudiante universitario de acuerdo a su respuesta. Los valores posibles son 1, 2, 3, 4 o 5. Las categorías de la variable respuesta presentan un orden intrínseco, por esta razón, para explicar su variabilidad es necesario el ajuste de un modelo de regresión logística ordenada.

\section{- Variables independientes}

Factores 1 a 4 del ACP: puntuaciones obtenidas por el método de Bartlett de los 4 factores extraídos mediante el análisis de componentes principales

Variable de control: Logaritmo natural del Producto Interno Bruto del país (USD) (Banco Mundial , 2016)

Variable de Control: Región donde se encuentra la Universidad. En un estudio previo realizado por este equipo utilizando esta misma base de datos se analizó la relación entre la intención emprendedora, actitudes emprendedoras personales, la motivación institucional y el apoyo familiar a la hora de emprender, concluyendo que la intención de emprender era significativamente mayor en las universidades del Norte (Colombia, Ecuador y México), que en las del Sur del continente (Brasil, Chile y Uruguay). . La Figura 3 muestra los resultados del análisis de conglomerados, observándose un agrupamiento de las universidades participantes del estudio de acuerdo a su posición geográfica en el continente. El cluster 1 (Sur) lo conformaron Universidades de Brasil, Chile y Colombia, mientras que en el cluster 2 se localizaron las Universidades de Colombia, Ecuador y México.

Figura 3 Análisis de conglomerados

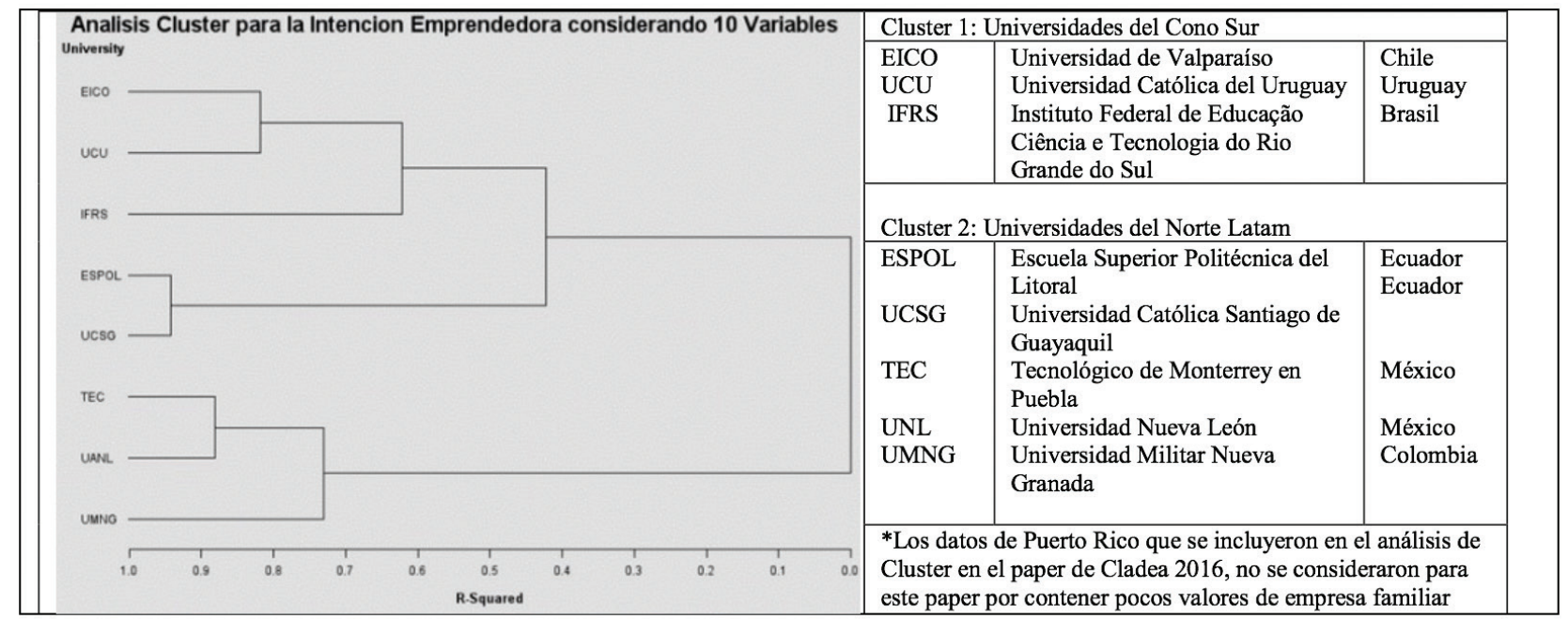

Para la obtención del objetivo final se desarrolló un modelo de regresión logística ordinal, donde la variable dependiente es el grado de intención emprendedora del estudiante universitario. Se probó primero que el modelo es adecuado para los datos de esta muestra, comparando la bondad de ajuste de un modelo solo con intercepto versus el modelo con todas las variables explicativas (Tabla 2). 
Tabla 2 Información sobre el ajuste del Modelo

\begin{tabular}{lllll}
\hline Modelo & $\begin{array}{l}-2 \text { Log de la } \\
\text { Verosimilitud }\end{array}$ & Chi-Square & df & Sig. \\
\hline Sólo intercepto & 1853,977 & & & \\
Final & 1559,052 & 294.925 & 6 &, 000 \\
\hline
\end{tabular}

Link function: Logit.

Se presenta la prueba de hipótesis del estudio: $\boldsymbol{H}_{\mathbf{0}}$ : el modelo es adecuado sólo con la constante.

$H_{1}$ : el modelo no es adecuado sólo con la constante.

Tabla 3 Bondad del ajuste

\begin{tabular}{l|l|l|l}
\hline & Chi-Square & df & Sig. \\
\hline Pearson & 2976,531 & 2966 &, 442 \\
Deviance & 1559,052 & 2966 & 1,000 \\
\hline
\end{tabular}

Link function: Logit.

Las hipótesis de la prueba son:

$H_{0}$ : el modelo se ajusta adecuadamente a los datos

$H_{1}$ : el modelo no se ajusta adecuadamente a los datos

Debido a que los p-valores de las pruebas son mayores que 0,05 , no se rechaza la hipótesis nula. Por tanto, el significado estadístico que resulta indica que el modelo con las variables introducidas se ajusta adecuadamente a los datos.

La Tabla 4 muestra, para este tipo de modelos, medidas equivalentes al coeficiente de determinación $\mathrm{R}^{2}$ de los modelos linealesque resumen la proporción de la variabilidad en la variable dependiente (intención emprendedora) asociada con los factores de predicción (variables independientes).Estos valores del pseudo-r cuadrado son muestras de la variabilidad explicada por el modelo, y en ellas se observa que el $\mathrm{R}^{2}$ Nagelkerke estima en un $34,9 \%$ tal variabilidad.
Debido a que el p-valor de la prueba es menor que 0,05 , se rechaza la hipótesis nula. Por tanto, el significado estadístico que resulta indica que el modelo con las variables introducidas mejora el ajuste de forma significativa respecto al modelo con sólo la constante.

En la siguiente tabla (Tabla 3) se presenta el test de chi-cuadrado de Pearsony el estadístico derivado de chi-cuadrado de desviación, el cual tiene por objeto comprobar si los datos observados son incompatibles con el modelo ajustado.

Tabla 4 Pseudo R-Square

\begin{tabular}{ll}
\hline Cox and Snell & .318 \\
Nagelkerke & .349 \\
McFadden & .159 \\
\hline
\end{tabular}

Link function: Logit.

La Tabla 5 muestra la estimación de los parámetros del modelo, sus errores estándares, la prueba chi-cuadrado de Wald de significación de cada variable y el intervalo de confianza del 95\% para cada parámetro. Se observa que todas las variables resultaron estadísticamente significativas considerando un nivel de significaron del 10\% (todos los valores-p fueron menores a 0,10 ). Los Factores 1 (Relacionamiento con la Empresa) y 3 (Motivación) fueron altamente significativos (valores-p < 0,001). Las variables de control 'Región' y 'PBI' (tomado como logaritmo natural) también resultaron altamente significativas (valores-p $=0,26$ y 0,006 $<0,05$ ). Los Factores 2 (Innovación, valor$\mathrm{p}=0,059>0,05)$ y 4 (Act.Personales, valor- $\mathrm{p}=0,091>0,05)$ fueron marginalmente no significativos al 5\% .

El modelo de regresión logística ordenada que corresponde a la salida de la Tabla 5 es:

(7) $\operatorname{Ln}\left(\frac{P(\text { Intencion Emprendedor } a \leq m)}{1-P(\text { Intencion Emprendedor } a \leq m)}\right)=$

$$
\tau_{m}-\beta_{1} \text { Factor } 1-\beta_{2} \text { Factor } 2-\beta_{3} \text { Factor } 3-\beta_{4} \text { Factor } 4-\beta_{5} \operatorname{Ln}(P B I)-\beta_{6}(\text { Region }=1)
$$

con $\mathrm{m}=1,2,3,4$ y 5

Despejando da como resultado la ecuación de probabilidad acumulada:

$$
\begin{aligned}
& P(\text { Intencion Emprendedora } \leq m) \\
& =\frac{1}{1+\exp \left[\left(\tau_{m}-\beta_{1} \text { Factor } 1-\beta_{2} \text { Factor } 2-\beta_{3} \text { Factor } 3-\beta_{4} \text { Factor } 4-\beta_{5} \operatorname{Ln}(P B I)-\beta_{6}(\text { Region }=1)\right]\right.}
\end{aligned}
$$

A partir de la Tabla 5 se puede concluir:

Los coeficientes estimados de la regresión logística ordenada indican el cambio esperado en la intención emprendedora del estudiante (medido en la escala de 'odd' o 'chance' de aumentar la categoría de intención emprendedora) por cambio unitario en el valor de la variable correspondiente. Podemos observar que los cuatro Factores que resultaron del análisis de Componentes Principales tuvieron un efecto positivo en la intención emprendedora (todos los coeficientes estimados fueron mayores de 0). De ellos el Factor 3 (Motivación, $\widehat{\beta_{3}}$ $=1,249$ ) tuvo el mayor impacto en la intención emprendedora (esto es, la intención emprendedora aumenta en promedio 1,249 unidades 
por cada incremento unitario del valor del Factor 3). El Factor 1 (Relacionamiento con la Empresa, $\widehat{\beta_{1}}=0,411$ ), Factor 2 (Innovación, $\widehat{\beta_{2}}=0,148$ ) y Factor 4 (Actitudes personales, $\widehat{\beta_{4}}=0,129$ ) le siguieron en orden de la magnitud del impacto en la intención emprendedora.

Las cuatro hipótesis del modelo teórico de investigación se verifican al 10\% de significación. Esto es, los factores definidos como 'Relacionamiento con la Empresa' (valor-p < 0,001), 'Innovación' (valor-p $=0,059$ ), 'Motivación' (valor-p < 0,001) y las 'Actitudes Personales' (valor-p $=0,091$ ) tienen un efecto significativo y positivo sobre la intención emprendedora de los estudiantes universitarios. Valores altos de estos factores están asociados a valores altos en la intención emprendedora. Considerando un nivel de significación del 5\%, solo los Factores 1 (Relacionamiento con la Empresa) y 3 (Motivación) resultaron significativos. El Factor 2 (Innovación) resulto marginalmente insignificantico al 5\%.
Se observó un efecto significativo de la Región sobre la intención emprendedora de los estudiantes universitarios (valor-p=0,026<0,05). El valor del parámetro estimado del efecto para la Región 1 (SUR) fue de $\widehat{\beta_{3}}=-0,520$. Esto es, $-0,520$ es el cambio esperado en la intención emprendedora (medida en la escala de 'chance') entre las regiones Sur (Región 1) y Norte (Región 2), manteniendo todas las otras variables constantes. En otras palabras, las chances de que un estudiante muestre una alta intención emprendedora son mayores en la Región Norte que en la Sur, siendo esta diferencia significativa a un nivel del 5\%. El PBI del país (tomado como logaritmo natural) resultó una variable de control significativa en el modelo (valor-p $=0,006$ ).

En conclusión, se observa que la intención emprendedora de los estudiantes universitarios es mayor cuando aumentan los valores que integran los Factores resultantes del ACP (Relacionamiento con la empresa, Innovación, Motivación, Actitudes Personales) y el estudiante pertenece a los países que integran la Región Norte del continente.

Tabla 5 Estimación de los parámetros

\begin{tabular}{|c|c|c|c|c|c|c|c|c|}
\hline & & \multirow{2}{*}{ Estimate } & \multirow{2}{*}{ Std. Error } & \multirow{2}{*}{ Wald } & \multirow{2}{*}{ df } & \multirow{2}{*}{ Sig. } & \multicolumn{2}{|c|}{ 95\% Confidence Interval } \\
\hline & & & & & & & Lower Bound & Upper Bound \\
\hline \multirow{4}{*}{ Threshold } & [Intención_Emprendedora $=1.0$ ] & $-21,587$ & 6,293 & 11,769 & 1 &, 001 & $-33,920$ & $-9,254$ \\
\hline & [Intención_Emprendedora = 2.0] & $-20,630$ & 6,289 & 10,760 & 1 & ,001 & $-32,956$ & $-8,304$ \\
\hline & [Intención_Emprendedora = 3.0] & $-19,036$ & 6,281 & 9,185 & 1 &, 002 & $-31,347$ & $-6,725$ \\
\hline & [Intención_Emprendedora = 4.0] & $-17,555$ & 6,275 & 7,827 & 1 & ,005 & $-29,853$ & $-5,257$ \\
\hline \multirow{7}{*}{ Location } & $\operatorname{Ln}(\mathrm{PBI})$ & $-1,784$ & ,647 & 7,593 & 1 &, 006 & $-3,053$ &,- 515 \\
\hline & $\begin{array}{l}\text { Factor } 1 \text { (Relacionamiento con la } \\
\text { empresa) }\end{array}$ & ,411 & ,089 & 21,370 & 1 &, 000 &, 237 & ,585 \\
\hline & Factor 2 (Innovación) &, 148 & ,078 & 3,572 & 1 &, 059 &,- 005 & ,302 \\
\hline & Factor 3 (Motivación) & 1,249 & ,085 & 214,615 & 1 &, 000 & 1,082 & 1,416 \\
\hline & Factor 4 (Act.Personales) &, 129 & ,076 & 2,853 & 1 & ,091 &,- 021 & ,278 \\
\hline & Región=1 (Sur) &,- 520 & ,234 & 4,965 & 1 &, 026 &,- 978 &,- 063 \\
\hline & Región=2 (Norte) & $0^{\mathrm{a}}$ & . & . & 0 & . & . & . \\
\hline
\end{tabular}

Link function: Logit.

\section{Comentarios finales}

La intención emprendedora en los estudiantes universitarios es un tópico central en la investigación en emprendedurismo. El principal aporte de esta investigación es proveer un nuevo ángulo a través del planteamiento de una ecuación matemática que permita cuantificar y predecir la intención emprendedora de un estudiante universitario a partir de los 4 factores y variables de control, que deberá ser probada en futuras investigaciones para analizar su validez en otras muestras. De la investigación se puede confirmar que el Factor 3 (Motivación, $\left.\widehat{\beta_{3}}=1,249\right)$ tuvo el mayor impacto en la intención emprendedora.

A su vez las cuatro hipótesis del modelo teórico de investigación se verifican al 10\% de significación. Esto es, los actores definidos como 'Relacionamiento con la Empresa' (valor-p < 0,001), 'Innovación' (valor-p = 0,059), 'Motivación' (valor-p < 0,001) y las 'Actitudes Personales' (valor-p $=0,091$ ) tienen un efecto significativo y positivo sobre la intención emprendedora de los estudiantes universitarios.
En nuestro estudio anterior se analizó el efecto de las regiones a través de un análisis de cluster. El presente estudio confirma el efecto significativo de la Región sobre la intención emprendedora de los estudiantes universitarios (valor-p=0,026 < 0,05). Esto es, los estudiantes de la región Norte de Latinoamérica (Ecuador, Colombia y México) tiene una probabilidad más alta de volverse emprendedores que los de la región Sur (Brasil, Chile y Uruguay).

Para futuras investigaciones se sugiere realizar un análisis crosscultural entre los países analizados, dado que el modelo del TBP sugiere que las normas subjetivas son antecedentes importantes de la intención empresarial que depende de la cultura nacional según las dimensiones culturales por país, donde se analicen los niveles tanto profesionales como organizacionales, y la forma de compartirla de una sociedad (Choia y Hofstede, 2016).

Limitaciones: la muestra analizada fue voluntaria de estudiantes que estaban realizando algún curso de emprendimiento de carreras empresariales e ingeniería y que sus familias tuvieran empresa familiar. 
Futuras investigaciones: consideramos interesante realizar un estudio en otras universidades o en carreras que no contengan cursos de emprendimiento para contrastar los resultados obtenidos.

\section{Agradecimientos}

Se agradece el aporte de la RLIE Red Latinoamericana de Innovación y Emprendimiento de CLADEA, que permitió generar los lazos para realizar la investigación, y en especial al TEC de Puebla que aportó el instrumento modificado por los investigadores, así como a los miembros de la RED que aportaron los datos de sus estudiantes: Margarita Herrera PhD y Lizbeth González PhD, Tecnológico de Monterrey en Puebla, México; Dra. Mónica Blanco Jiménez, $\mathrm{PhD}$, Universidad Autónoma de Nuevo León; Leslie Bridshaw Araya Mag, Universidad de Valparaíso, Chile; Carolina Ortiz Riaga Mag, Universidad Militar Nueva Granada, Colombia; Shana Sabbado Flores $\mathrm{PhD}$ y Claudio Vinícius Silva Farias PhD, Instituto Federal de Educação Ciência e Tecnologia do Rio Grande do Sul, Brasil; Nancy Wong Laborde PhD, Carmen Padilla Lozano, PhD (c) y Danny Arévalo Avecillas PhD (c), Universidad Católica Santiago de Guayaquil, Ecuador ; María Paulina Brito Ochoa MBA, Escuela Superior Politécnica del Litoral, Ecuador; Gisela I. Carrero Morales PhD y Carmen L. Rivera Hernández MBA, Universidad Interamericana de Puerto Rico Recinto de Bayamón.

\section{Bibliografía}

Ajzen, I. (1991). Theory of Planned Behavior. Organization Behavior and Human Decision Processes, 50(2), 179-211. doi:10.1016/j.obhdp.2010.11.001

Ajzen, I. (2002). Perceived behavioral control, self-efficacy, locus of control, and the theory of planned behavior. Journal of Applied Social Psychology, 32(1), 1-20. doi:10.1111/j.1559-1816.2002.tb00236.x

Autio, E., Kenney, M., Mustar, P., Siegel, D., \& Wright, M. (2014). Entrepreneurial innovation: The importance of context. Research Policy, 1-11. doi:10.1016/j.respol.2014.01.015

Banco Mundial . (2016). El Banco Mundial - Datos . Retrieved 06 2, 2016, from http://datos.bancomundial.org/region/LAC

Bergmann, H., Hundt, C., \& Sternberg, R. (2016). What makes student entrepreneurs?- On the relevance (and irrelevance) of the university and the regional context for student start-ups. Small Business Economics. doi:10.1007/s11187-016-9700-6

Bonomo, A., Krauss, C., \& Volfovicz, R. (2017). "La intención emprendedora del estudiante universitario que proviene de una empresa familiar en seis países latinoamericanos". Proceeding 52a Asamblea Anual de CLADEA Rompiendo Paradigmas: Anticipando el Futuro de la Educación Global, 17 al 19 octubre 2017 disponible en proceeding. Riverside, California -USA: CLADEA. Retrieved 07 06, 2018, from http://grupoinolsa.com/ojs3/index.php/procceding_cladea2017/article/view/184/150
Brändle, L., Berger, E. S., \& Kuckertz, A. (2018). I am what I amHow nascent entrepreneurs' social indentity affects their entrepreneurial self-efficacy. Journal of Business Venturing Insights, 9, 17-23. doi:10.1016/j.jbvi.2017.12.001

Brännback, M., Carsrud, A., \& Schulte, W. D. (2008). Exploring the role of Ba in family business context. VINE, 38(1), 104-117.

Choia, K. S., \& Hofstede, G. J. (2016). A cross-cultural comparative analysis of small group collaboration using mobile twitter. Computer in Human Behavior, 65, 308-318. doi:10.1016/j.chb.2016.08.043.

Criaco, G., Sieger, P., Wennberg, K., Chirico, F., \& Minola, T. (2017). Parents' performance in entrepreneurship as a "double-edged sword" for the intergenerational transmission of entrepreneurship. Small Business Economics, 1-24. doi:10.1007/s11187-017-9854-x

Diéguez-Soto, J., Garrido-Moreno, A., \& Manzaneque, M. (2018). Unravelling the link between process innovation inputs and outputs: The moderating role of family management. Journal of Family Business Strategy, 9, 114-127. doi:10.1016/j.jfbs.2017.11.007

Fayolle, A. (2013). Personal views on the future of entrepreneurship education. Entrepreneurship and Regional Development., 25((7/8)), 692-701. doi:10.1080/08985626.2013.821318

González, L. A., \& Herrera, M. (2017). La intención del sucesor en la continuidad de la empresa familiar. (pp. 1-15). San Bernardino. Los Angeles: CLADEA. Retrieved from http://grupoinolsa.com/ojs3/index.php/procceding_cladea2017/article/view/186/152

Gubik, A., \& Farkas, S. (2016). Student Entrepreneurship in Hungary: Selected Results Based on Guesss Survey. Entrepreneurial Business and Economics Review, 4(4), 123-139. doi:10.1111/j.1467-8586.1990. tb00290.x.

GUESSS. (2013). Global University Entrepreneurial Spirit Students'Survey Student Entrepreneurship Across the Globe: A Look at Intentions and Activities. Retrieved 4 15, 2017, from http://www.guesssurvey.org/PDF/2013/GUESSS_INT_2013_REPORT.pdf

Kakouris, A., \& Georgiadis, P. (2016). Analysing entrepreneurship education: a bibliometric survey pattern. Journal Of Global Entrepreneurship Research, 6(1), 1. doi:10.1186/s40497-016-0046-y

Karabulut, E. O., \& Dogan, P. K. (2018). Investigation of Entrepreneurship Trends and General Competency Levels of University Student Studying at Faculty of Sports Sciences. Journal of Education and Training Studies, 6(4), 212-220. doi:10.11114/jets.v6i4.3128

Krauss, C., Bonomo, A., Volfovicz, R., Bonbois, M., Herrera, M., González, L., . . Rivera, C. (2016). Una división Norte-Sur en la Intención Emprendedora del estudiante universitario: El caso de 8 universidades. CLADEA. Medellín. 
Krueger, N. F., Reilly, M. D., \& Carsrud, A. L. (2000). Competing Models of Entrepreneurial Intentios. Journal of Business Venturing, 15, 411-432.

Liñán, F., \& Chen, Y. (2009). Development and cross-cultural application of a specific instrument to measure entrepreneurial intentions. Entrepreneurship: Theory And Practice, 33(3), 593-617.

Luan, C.-J., Chen, Y.-Y., Huang, H.-Y., \& Wang, K.-S. (2017). CEO succession decision in family businesses - A corporate governance perspective. Asia Pacific Management Review. doi:https://doi-org. proxy.timbo.org.uy:88/10.1016/j.apmrv.2017.03.003

Maes, J., Leroy, H., \& Sels, L. (2014). Gender differences in entrepreneurial intentions: A TPB multi-group analysis at factor and indicator level. European Management Journal, 32, 784-794. doi:https://doi. org/10.1016/j.emj.2014.01.001

McClelland, D. C., \& Watson, R. I. (1973). Power motivation and risk-taking behavior. journal of Personality, 41(1), 121-139. doi:10.1111/j.1467-6494.1973.tb00664.x

McKelvey, R. D., \& Zavoina, W. (1975). A statistical model for the analysis of ordinal level dependent variables. Journal of mathematical sociology, 4(1), 103-120. doi:10.1080/0022250X.1975.9989847
Miller, S. P. (2014). Next-generation leadership development in family businesses: the critical roles of shared vision and family climate. Frontiers In Psychology, 51-14., 51(14). doi:10.3389/fpsyg.2014.01335

Navarro de Granadillo, K. (2008). Estado actual de la investigación sobre la gestión del conocimiento en empresas familiares. Revista de Ciencias Sociales, XIV(1), 30-45. doi:1315-9518

Rondi, E., De Massis, A., \& Kotlar, J. (2017). Unlocking innovation potential: A typology of family business innovation postures and the critical role of the family system. Journal of Family Business Strategy, 1-11. doi:10.1016/j.jfbs.2017.12.001

Schott, T., Kew, P., \& Cheraghi, M. (2015, abril 27). Future Potential. A GEM perspective on youth entrepreneurship 2015. Retrieved from Gemconsortium: http://www.gemconsortium.org/about/news/18

Shirokova, G., Osiyevskyy, O., \& Bogatyreva, K. (2016). Exploring the intention-behavior link in student entrepreneurhip: Moderating effects of individual and environmental characteristics. European $\mathrm{Ma}$ nagement Journal, 34(4), 386-399. doi:10.1016/j.emj.2015.12.007

Yurtkoru, S., Kuscu, Z. K., \& Doganay, A. (2014). Exploring the antecedents of entrepreneurial intention on Turkish university students. Procedia-Social and Behavior Sciences(10th International Strategic Management Conference 2014), 841-850. doi:10.1016/j.sbspro.2014.09.093 


\section{Anexo:}

\begin{tabular}{lll}
\hline Resultados del Análisis Factorial de Factor 1: & Valor Propio & Varianza Explicada \\
\hline Factor 1. Relacionamiento con la empresa & & 61,228 \\
\hline 44. Relación con la empresa & 0,749 & 0,904 \\
45. Relación con los propietarios & 0,814 \\
46. Sacrificio por la empresa & 0,865 \\
47. Acciones para mejorar la empresa & 0,871 \\
48. Carrera relacionada con empresa familiar & 0,704 \\
49. Experiencia en otra empresa & 0,578 \\
50. Dirigir la empresa & 0,822 & \\
52. Disfruta trabajar en la empresa & 0,814 & \\
\hline
\end{tabular}

$\left(\mathrm{KMO}=0,906 ; X^{2} \operatorname{Bartlett}(28)=4226,665 ; \mathrm{p}<0.000\right.$; Varianza total explicada 61,228$)$

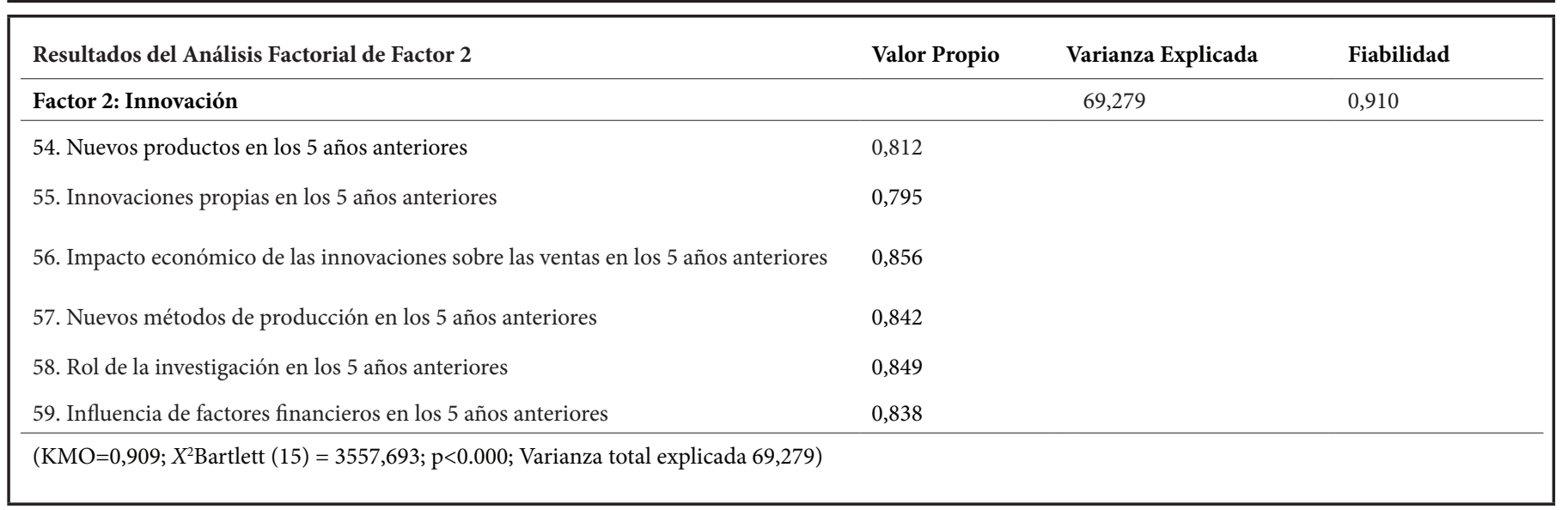

\begin{tabular}{|c|c|c|c|}
\hline Resultados del Análisis Factorial de Factor 3: & Valor Propio & Varianza Explicada & Fiabilidad \\
\hline Factor 3: Motivación para emprender & & 52,267 & 0,814 \\
\hline 23.d La estimulación & 0,704 & & \\
\hline 23.e La autodirección & 0,787 & & \\
\hline 23. i Apoyo familiar para emprender & 0,734 & & \\
\hline \multicolumn{4}{|c|}{$\left(\mathrm{KMO}=0,858 ; X^{2}\right.$ Bartlett $(15)=5770,262 ; \mathrm{p}<0.000 ;$ Varianza total explicada 52,267 $)$} \\
\hline
\end{tabular}

\begin{tabular}{lll}
\hline Resultados del Análisis Factorial de Factor 4 & Valor Propio & Varianza Explicada \\
\hline Factor 4: Actitud Personal: Poder Hedonismo & & 68,716 \\
\hline 23.a. El poder & 0,829 & \\
23.c El placer & 0,829 & \\
$\left(\mathrm{KMO}=0,500 ; X^{2}\right.$ Bartlett $(1)=501,249 ; \mathrm{p}<0.000 ;$ Varianza total explicada 68,716$)$ & & \\
\hline
\end{tabular}


